\title{
Measures of space-time nonseparability of electromagnetic pulses
}

\author{
Yijie Shen $\odot,{ }^{1, *}$ Apostolos Zdagkas $\odot,{ }^{1}$ Nikitas Papasimakis, ${ }^{1}$ and Nikolay I. Zheludev ${ }^{1,2}$ \\ ${ }^{1}$ Optoelectronics Research Centre and Centre for Photonic Metamaterials, University of Southampton, \\ Southampton SO17 1BJ, United Kingdom \\ ${ }^{2}$ Centre for Disruptive Photonic Technologies, School of Physical and Mathematical Sciences and The Photonics Institute, \\ Nanyang Technological University, Singapore 637378, Singapore
}

(Received 27 July 2020; revised 17 December 2020; accepted 21 January 2021; published 12 March 2021)

\begin{abstract}
Electromagnetic pulses are typically treated as space-time (or space-frequency) separable solutions of Maxwell's equations, where spatial and temporal (spectral) dependence can be treated separately. In contrast to this traditional viewpoint, recent advances in structured light and topological optics have highlighted the nontrivial wave-matter interactions of pulses with complex space-time nonseparable structure, as well as their potential for energy and information transfer. A characteristic example of such a pulse is the "flying doughnut" (FD), a space-time nonseparable few-cycle pulse with links to toroidal and nonradiating (anapole) excitations in matter. Here, we propose a quantum-mechanics-inspired methodology for quantitatively characterizing space-time nonseparability in structured pulses. In analogy to the mathematics of nonseparability in quantum mechanics, we introduce the concept of space-spectrum nonseparable states to describe the space-time nonseparability of a classical electromagnetic pulse and apply the state tomography method to reconstruct the corresponding density matrix. Using the example of the FD pulse, we calculate the fidelity, concurrence, and entanglement of formation as their quantitative measures, and we demonstrate that such properties dug out from quantum mechanics can quantitatively characterize the spatiotemporal evolution of general structured pulses. Our results highlight the potential of space-time nonseparable pulses as information carriers and facilitate their deployment in information transfer and cryptography applications.
\end{abstract}

DOI: 10.1103/PhysRevResearch.3.013236

\section{INTRODUCTION}

The generation, diagnostics, and applications of spatiotemporal electromagnetic pulses have attracted growing interest from diverse research communities, including metamaterials [1,2], communications [3], particle acceleration [4,5], laser machining [6-8], and nonlinear and topological photonics [9-12]. Typically, such pulses are treated as space-time (or, equivalently, space-frequency) separable solutions of Maxwell's equations, which can be expressed as a product of a spatial mode and a temporal (or spectral) function, following the traditional separation of variables for solving partial differential equations. Since it is widely endorsed that electromagnetic pulses are practically space-time separable, the space-time nonseparability (STNS) is usually ignored for simplification, but STNS actually exists in any pulse [13]. STNS can play a major role in the propagation dynamics [14-16], nondiffraction [17-19], and anomalous refraction [20] of pulses. A simple but important consequence of STNS is the separation of pulse frequency components upon prop-

\footnotetext{
*y.shen@soton.ac.uk

Published by the American Physical Society under the terms of the Creative Commons Attribution 4.0 International license. Further distribution of this work must maintain attribution to the author(s) and the published article's title, journal citation, and DOI.
}

agation leading to distinct effects, such as isodiffraction and isodivergence [14]. The parameters of these monochromatic components can hence define the overall shape and characteristics of these pulses, such as evolution of transverse spectral distribution and carrier envelope phase, which can then be tailored for efficient control of attosecond processes [21], chemical reactions [22] and ultrafast pump-probe experiments [23].

Space-time nonseparable exact solutions of Maxwell's equations have been known to exist for a long time. In 1983, Brittingham proposed the localized (e.g., nondiffracting) solutions to Maxwell's equations termed "focus wave modes" [24], as typical examples of STNS pulses. Soon after, Ziolkowski generalized the STNS solutions to the scalar wave equation with moving complex sources [25] and proposed that superpositions of such pulses lead to finiteenergy pulses termed "electromagnetic directed-energy pulse trains" (EDEPTs) [26]. A series of methods for constructing and designing localized waves and nondiffracting waves then emerged based on the EDEPT method [27-30]. Special cases of Ziolkowski's solutions were studied by Hellwarth, Nouchi, and co-workers, who found closed-form expressions that describe single-cycle finite-energy STNS solutions to the homogeneous Maxwell's equations, the family of which includes both linearly polarized pulses, termed "pancakes" [31], and pulses of toroidal symmetry, termed "flying doughnuts" (FDs) [32]. The exotic FD pulses hold promise as novel information and energy carriers, particularly in the contexts 
of nonradiating anapole configurations [33,34], topological information transfer [35], probing ultrafast light-matter interactions [36], and toroidal excitations in matter [37,38]. Recently, it was demonstrated that the FD pulses can be generated by tailored metamaterials which can convert traditional few-cycle pulses into STNS pulses [39,40].

Nonseparability is also a quintessential property of quantum entanglement between particles; for example, an entangled particle pair state cannot be expressed as the product of two single-particle states, and as a result the measurement of one particle affects the measurement outcome of another [41]. A typical example is the polarization-entangled photon pair where the polarization states of the two photons are nonseparable. Over the past century, an extended toolbox has been developed that allows us to quantify the nonseparability of entangled states, including state tomography, density matrix, fidelity, linear entropy, and concurrence, etc. [42,43]. Recently, the tools of quantum mechanics were constructively applied not only to quantum physics but also to classical optics [44-48]. For example, the concept of quantum coherent states can be used to describe complicated laser modes [49-53]. The quantum Bell's measure was also applied in classical optical coherence [54]. Many classical analogs of quantum states were realized in vortex beams such as Laughlin states [55] and Schrödinger's cat states [56]. Moreover, vector vortex beams with space-polarization nonseparability can simulate spin-orbital angular momentum entanglement [57-60]. Recently, a measure of entanglement, the Schmidt number, was used to characterize nondiffracting properties in optical wave packets [61]. These useful applications of quantum mechanics in classical optics have motivated the development of novel methods in (tele)communications [62-64], cryptography [65], and metrology [66-68].

In this paper, we draw on the mathematic analogies between classical STNS and quantum entanglement and exploit mathematic tools from quantum mechanics to measure the STNS of classical pulses. In particular, we present a state

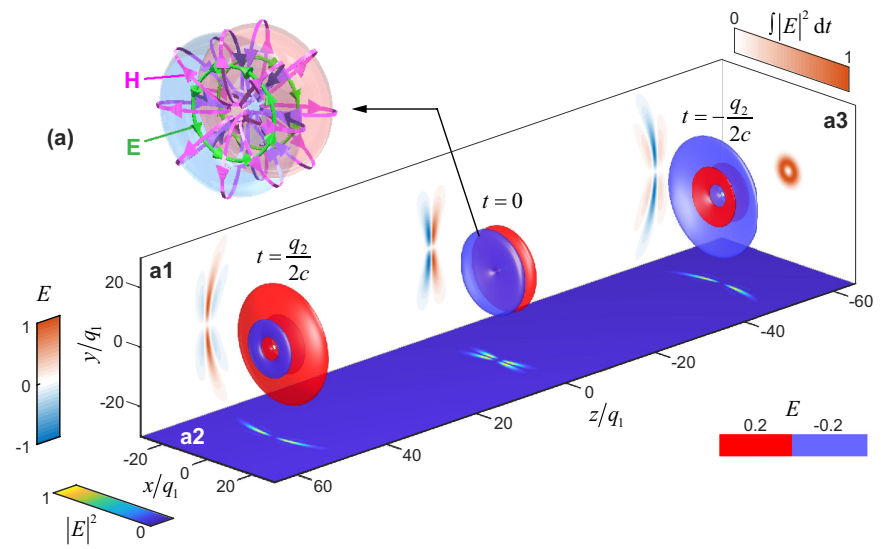

tomography approach to reconstruct the density matrix of space-time nonseparable states. We apply our approach to general pulses with prescribed STNS, such as the FD pulse and superposed Laguerre-Gaussian (LG) modes. We demonstrate that the STNS measures introduced here allow us to quantitatively characterize the evolution of the pulse's spatiospectral structure upon propagation. This work introduces a new toolkit for characterizing a general family of pulses with various degrees of intrinsic space-time coupling and provides new perspectives to study their spatiotemporal structure and propagation dynamics. The approach proposed here will lead to insights into light-matter interactions with ultrafast structured pulses and extend applications in spectroscopy, cryptography, and communications.

\section{DYNAMICS OF FLYING DOUGHNUT PULSE}

FDs are few-cycle doughnutlike electromagnetic pulses with a toroidal configuration of electric and magnetic fields. They exist both as transverse electric (TE) and as transverse magnetic (TM) pulses. In the former case, the electric and magnetic fields are given by [32]

$$
\begin{array}{r}
\boldsymbol{E}=E_{\theta} \hat{\boldsymbol{\theta}}=-f_{0} i \sqrt{\frac{\mu_{0}}{\varepsilon_{0}}} \frac{r\left(q_{1}+q_{2}-2 i c t\right)}{\left[r^{2}+\left(q_{1}+i \tau\right)\left(q_{2}-i \sigma\right)\right]^{3}} \hat{\boldsymbol{\theta}}, \\
\boldsymbol{H}=H_{r} \hat{\boldsymbol{r}}+H_{z} \hat{\boldsymbol{z}}=f_{0} i \frac{r\left(q_{2}-q_{1}-2 i z\right)}{\left[r^{2}+\left(q_{1}+i \tau\right)\left(q_{2}-i \sigma\right)\right]^{3}} \hat{\boldsymbol{r}} \\
-f_{0} \frac{r^{2}-\left(q_{1}+i \tau\right)\left(q_{2}-i \sigma\right)}{\left[r^{2}+\left(q_{1}+i \tau\right)\left(q_{2}-i \sigma\right)\right]^{3}} \hat{\boldsymbol{z}},
\end{array}
$$

where $\sigma=z+c t, \tau=z-c t, f_{0}$ is a normalization constant, $\left(q_{1}, q_{2}\right)$ represent the effective wavelength and Rayleigh range, respectively, and $(\hat{\boldsymbol{r}}, \hat{\boldsymbol{\theta}}, \hat{\boldsymbol{z}})$ are the three normalized basis vectors of cylindrical coordinates. In particular, the value of the ratio $q_{2} / q_{1}$ indicates whether the pulse is well collimated $\left(q_{2} / q_{1} \gg 1\right)$ or strongly focused. In the TE mode, the electric

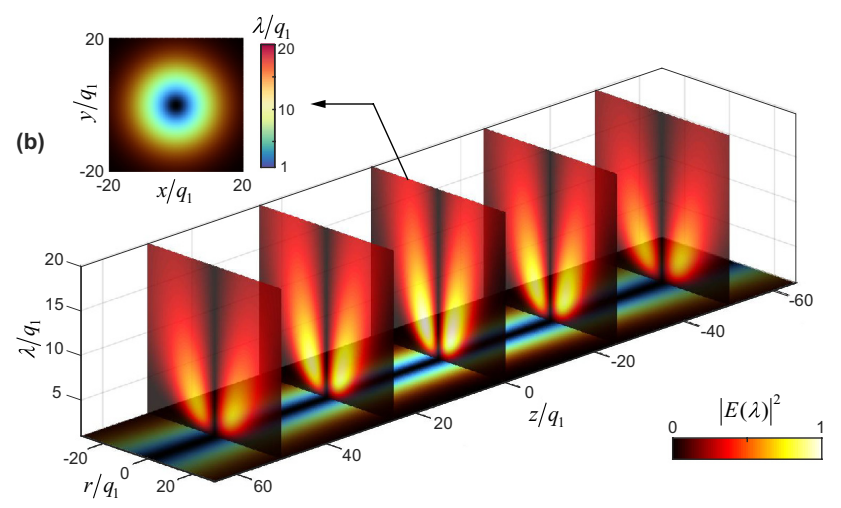

FIG. 1. (a) Spatiotemporal structure of the FD pulse: The spatial isosurfaces of the electric field $E(t, r, z)$ of the TE FD at different times of $t=0$ and $\pm q_{2} /(2 c)$, at amplitude levels of $E= \pm 0.2$. (a1) The $y-z$ cross section of the instantaneous electric field $E$ of the FD pulse at $x=0$ for $t=0$ and $\pm q_{2} /(2 c)$. (a2) The $x$ - $z$ cross section of the electric field intensity $|E|^{2}$ of the FD pulse at $x=0$, at $t=0$ and $\pm q_{2} /(2 c)$. (a3) The $x-y$ cross section of the electric field intensity of the FD pulse integrated over all times at the $z=0$ plane $\int_{-\infty}^{\infty}|E(t, r, 0)|^{2} d t$. The inset at top left shows the electromagnetic vector structure of the FD pulse at the focus $(z=0)$. (b) Spectral structure of the FD pulse: The slices in the $r-\lambda$ domain show $|\widetilde{E}(\lambda, r, z)|^{2}$ of the spectral components of the FD pulse at propagation distances $z$. The color map in the $r$ - $z$ plane shows a false-color image constructed by the distribution of monochromatic components at the corresponding radial position; the inset shows a similar false-color image in $x-y$ plane. 

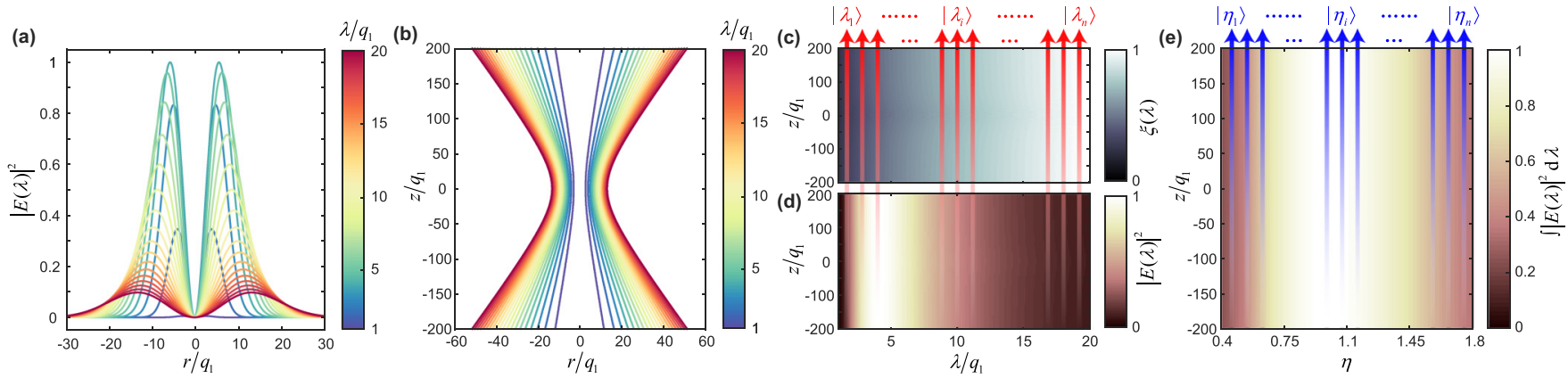

FIG. 2. (a) Profiles of radial distribution of normalized intensity $I(\lambda, r, z)=|\widetilde{E}(\lambda, r, z)|^{2} / \max _{(r, \lambda)}\left[|\widetilde{E}(\lambda, r, z)|^{2}\right]$ of different spectral components of the FD pulse at the focus $(z=0)$. Lines of different color represent monochromatic components of different wavelengths. (b) The color-coded traces of the radial positions where the intensity $I(\lambda, r, z)$ reaches its maximum for different wavelengths of the FD pulse. (c) Ratio $\xi(\lambda, z)=r_{\lambda} / r_{\lambda_{n}}$, where $r_{\lambda}$ is the radial position of the peak of the intensity $I(\lambda, r, z)$ of the monochromatic component at wavelength $\lambda$. Here, the radius $r_{\lambda_{n}}$ is used for normalization and corresponds to the position of peak intensity for a given wavelength $\lambda_{n}$ of the FD pulse. (d) Peak value of the intensity, $I_{\mathrm{m}}(\lambda, z)=I\left(\lambda, r_{\lambda}, z\right)$, for each monochromatic component of the FD pulse as a function of propagation distance $z$. (e) The normalized total field $I_{0}(r, z)=\int I(\lambda, r, z) d \lambda / \max _{r}\left[\int I(\lambda, r, z) d \lambda\right]$ plotted versus the value of $\eta(r, z)=r / r_{\text {max }}(z)$ at each propagation distance $z$, where $r_{\max }(z)$ is the radius at which $I_{0}(r, z)$ reaches its maximum. Note the isodiffraction property: $\xi(\lambda),\left|\widetilde{E}_{\mathrm{m}}(\lambda)\right|^{2}$, and $I(\eta)$ do not depend on $z$. The red and blue arrows demonstrate the positions of spectral and spatial states. Spectral states are represented by the trajectories of the electric field intensity maxima of the various monochromatic components, i.e., $r_{\lambda}(z)$, and spatial states are represented by the trajectories of prescribed positions $r(z)$ fulfilling the prescribed radial ratios of $\eta_{i}=r / r_{\max }(z)$.

field is azimuthally polarized with no longitudinal or radial components, whereas the magnetic field is oriented along the radial and longitudinal directions with no azimuthal component [see Fig. 1(a)]. Two different pulses can be constructed respectively from the real and imaginary parts of the complex electromagnetic fields of Eqs. (1) and (2), both types of which are exact solutions to Maxwell's equations. The real part is single cycle in the electric field and $1 \frac{1}{2}$ cycle in the magnetic field at the focus $(z=0)$, while the imaginary part is $1 \frac{1}{2}$ cycle in the electric field and single cycle in the magnetic field at the focus. Thus the real part is referred to as the single-cycle pulse, and the imaginary one is referred to as the $1 \frac{1}{2}$-cycle pulse. Upon propagation, the single-cycle ( $1 \frac{1}{2}$-cycle) pulse transforms to the $1 \frac{1}{2}$-cycle (single-cycle) pulse due to the Gouy phase shift [69]. The propagation dynamics of a singlecycle FD pulse $\left(q_{2}=100 q_{1}\right)$ are revealed by the isosurfaces of the electric field at various times in Fig. 1(a). Away from the focus $\left(z= \pm q_{2} / 2\right)$, the pulse displays $1 \frac{1}{2}$ cycle composed of a central bright doughnut and two darker toroidal lobes. At the focus $(z=0$ and $t=0)$, the pulse is single cycle with two equal-amplitude doughnuts corresponding to the two half cycles of the pulse.

\section{SPACE-SPECTRUM NONSEPARABLE STRUCTURE}

Due to its spatiotemporal structure, the FD pulse exhibits a frequency spectrum $\widetilde{E}(\lambda, r, z)$ with an exotic spatial distribution over a very broad spectral band [32]. Generally, all temporal properties can be fully characterized in the spectral domain; thus the STNS property can be equivalently interpreted by space-spectrum nonseparability, and the two terms will be used here interchangeably. The spatially dependent frequency spectrum of the FD pulse at various propagation distances is depicted in Fig. 1(b), which can be obtained by the closed-form expression of Fourier transforms of the FD pulse presented in our previous works [70]. Here, the short-wavelength (bluish) components are always tightly confined close to the center of the doughnut, while the long-wavelength (reddish) components are located at the periphery of the pulse [see the inset in Fig. 1(b)]. The spatial normalized intensity distribution, $I(\lambda, r, z)=|\widetilde{E}(\lambda, r, z)|^{2} / \max _{(r, \lambda)}\left[|\widetilde{E}(\lambda, r, z)|^{2}\right]$, of monochromatic components of different wavelengths $\lambda_{i}(i=$ $1,2, \ldots, n)$ is depicted in Fig. 2(a). The STNS in the FD pulse manifests as isodiffraction [71], ensuring that in each transverse plane (normal to the propagation direction) the spatial profiles of intensity for every frequency component scale along the trajectory of the beam in the same way. To illustrate the isodiffracting nature of the FD pulse, we trace the radial position, $r_{\lambda_{i}}$, of the peak of the intensity of each wavelength upon propagation in Fig. 2(b), $I\left(\lambda_{i}, r_{\lambda_{i}}, z\right)=$ $\max _{r}\left[I\left(\lambda_{i}, r, z\right)\right]$. We introduce the dimensionless ratio $\xi=$ $r_{\lambda_{i}} / r_{\lambda_{n}}$ of each trace, where the position of peak intensity of each monochromatic component is normalized to that of a given component at wavelength $\lambda_{n}$. In contrast to the radial positions of peak intensity [Fig. 2(b)], the ratio $\xi$ of each monochromatic component is propagation invariant [Fig. 2(c)]. A similar propagation-invariant picture can be seen for the peak intensity value, $I_{\mathrm{m}}(\lambda, z)=I\left(\lambda, r_{\lambda}, z\right)$, of various wavelengths [Fig. 2(d)]. To investigate the evolution of the transverse profile of total electric field intensity (integrated over the wavelength components), we introduce normalized radial positions $\eta=r / r_{\max }(z)$, where $r_{\max }(z)$ is the position of the maximum of the total electric field intensity in the transverse plane at propagation distance $z$. As shown in Fig. 2(e), the normalized total intensity profile, $I_{0}(\eta, z)=$ $\int I(\lambda, \eta, z) d \lambda / \max _{r}\left[\int I(\lambda, \eta, z) d \lambda\right]$, versus the normalized radius $\eta$ is also $z$ independent.

The introduction of the radial position ratios $\xi(\lambda)$, normalized radial coordinates $\eta$, and normalized electric field intensities $I_{\mathrm{m}}(\lambda)$ and $I_{0}(\eta, z)$ allows us to highlight the propagation-invariant characteristics of isodiffracting pulses, such as the FD. Indeed, in isodiffracting pulses, $\xi(\lambda), I_{\mathrm{m}}(\lambda)$, 
and $I_{0}(\eta)$ do not depend on the propagation distance $z$. In contrast, a generic polychromatic beam (e.g., a wideband superposed LG beam) is not expected to exhibit such propagation-invariant properties. Based on these properties, we can introduce two sets of states to describe STNS in broadband beams and pulses: (1) Spectral states $\left|\lambda_{i}\right\rangle(i=$ $1,2, \ldots, n)$ are (monochromatic) states of light of defined wavelength $\lambda_{i}$ and with defined radial position $\left(r_{\lambda_{i}}\right)$ of peak intensity; (2) spatial states $\left|\eta_{i}\right\rangle$ are (generally polychromatic) states of light located at the position with defined radial ratio of $\eta_{i}=r / r_{\max }$, where $r_{\max }$ is the radial position at which the total intensity of the light field (e.g., the broadband beam or pulse) reaches its maximum. Based on the prior theory in Ref. [47], here we can apply Dirac notation to the spectral and spatial states because the representations of the two states are both spatially distributed functions and the eigenvectors are expressed in the infinite-dimensional Hilbert space. Generally, the positions of the spectral and spatial states depend on propagation distance $z$. For convenience, we can also use the normalized radial position of $\eta=r / r_{\max }$. Thus, at a transverse plane at propagation distance $z$, we can represent spectral state $\left|\lambda_{i}\right\rangle$ by the peak intensity $I_{\mathrm{m}}\left(\lambda_{i}, z\right)$ at $\eta_{\lambda_{i}}(z)=$ $r_{\lambda_{i}}(z) / r_{\max }$. Similarly, a spatial state $\left|\eta_{i}\right\rangle$ can be represented by the intensity value $I_{0}\left(\eta_{i} r_{\max }, z\right)$ at normalized radial position $\eta_{i}$. The locations of spatial and spectral states, $\eta_{i}$ and $\eta_{\lambda_{i}}(z)$, respectively, define trajectories in the $\eta-z$ plane (see Fig. 3). For an arbitrary polychromatic beam, the trajectories of $\eta_{i}$ are always vertical lines in this plane, while $\eta_{\lambda_{i}}(z)$ can follow arbitrary trajectories. However, for an ideal isodiffracting pulse, both spectral and spatial states are represented by vertical trajectories reflecting the propagation invariance of the spatial and spectral intensity profile. Moreover, here, we choose sets of states in such a way that spatial and spectral states are perfectly coincident; that is, $\eta_{\lambda_{i}}(z)=\eta_{i}$ for isodiffracting pulses. The introduction of spatial and spectral sets of states allows us to distinguish apparently similar broadband waves. As an example, we consider two doughnutlike pulses with different STNS, the FD pulse and a wideband LG beam. Both pulses exhibit toroidal topology [see Figs. 3(a) and 3(b)] and a similar wideband spectrum, but they exhibit very different spatiospectral structure and propagation dynamics as illustrated by the corresponding spatial and spectral states. For the FD pulse, the spectral states are coincident with the corresponding spatial states upon propagation, as Fig. 3(c) shows. In contrast, the wideband LG beam is constructed by a set of monochromatic LG modes (see details of the wideband LG beam construction in Note 1 of the Supplemental Material [72]). As a result, the corresponding spectral and spatial states are naturally separated [see Fig. 3(d)], and the spatiospectral structure of the beam varies dramatically as it propagates. For example, at the focus, long-wavelength components are located close to the axis of the beam [Fig. 3(d1)], whereas away from the focus they move to the periphery of the beam [Fig. 3(d2)]. The difference between the isodiffracting FD and the broadband LG beam can be emphasized further in the $\eta-z$ plane. Here, as expected, the spectral states of the FD pulse [Fig. 3(e)] are $z$ invariant and coincident with the corresponding spatial states. On the other hand, the profile of the wideband LG beam [Fig. 3(f)] suffers substantial distortion as illustrated by the trajectories of the spectral states. This is

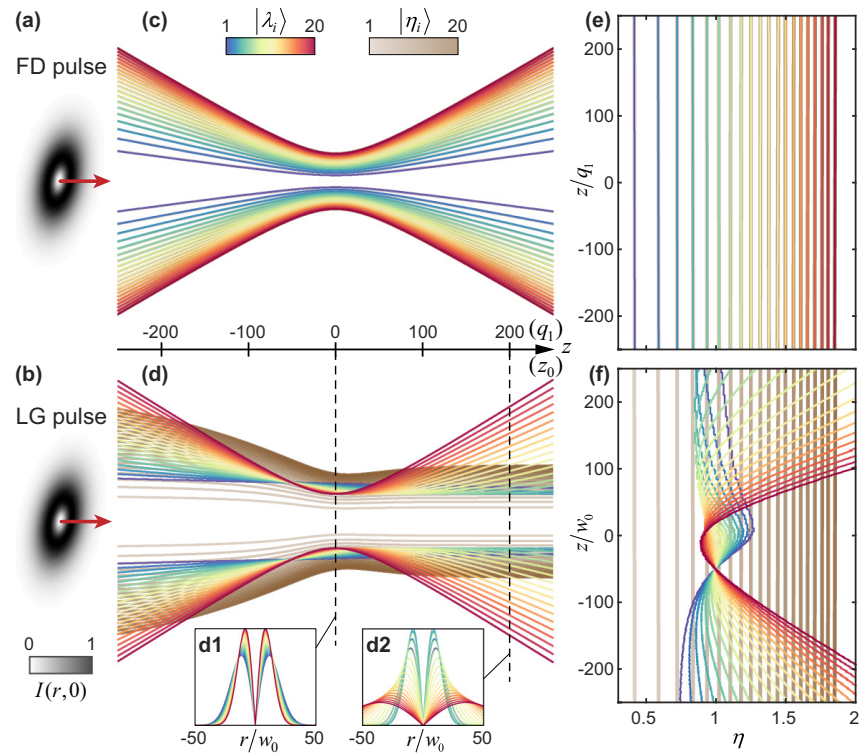

FIG. 3. (a) and (b) The transverse intensity patterns $\left.I_{0}(r)\right|_{z=0}$ of the FD pulse (a) and a wideband LG beam (see Note 1 of the Supplemental Material [72]) (b). (c) and (d) The propagation profiles of spectral $\left(\left|\lambda_{i}\right\rangle\right)$ and spatial $\left(\left|r_{i}\right\rangle\right)$ states of the FD beam (c) and wideband LG beam (d). The two insets in (d) show the spatial profiles of different wavelength components of the wideband LG beam at two different propagation distances, $z=0$ (d1) and $z=200 z_{0}(\mathrm{~d} 2)$, respectively, where $z_{0}$ is $1 / 100$ of the Rayleigh length (averaged over all monochromatic components of the beam). (e) and (f) The $\eta-z$ map of spectral and spatial states of the FD beam (e) and wideband LG beam (f). Note that the LG beam experiences dramatic distortion upon propagation, whereas the FD pulse profile remains invariant owing to its isodiffracting nature. In this illustration, we have considered 20 different spectral and spatial states, $\left|\lambda_{i}\right\rangle$ and $\left|\eta_{i}\right\rangle$ ( $i=1,2, \ldots, 20)$, where wavelength values are set as $\lambda_{i}=i q_{1}$ and radial ratios are selected as $\eta_{i}=r_{\lambda_{i}} / r_{\max }$.

a direct result of the noncoincidence of spectral and spatial states.

To quantify STNS in doughnutlike pulses, we interpret the problem as a measurement of an entanglementlike system, i.e., the nonseparability of two classical spatial fields [47]. In our implementation, the classical fields of the spectral state $\left|\lambda_{i}\right\rangle$ and the spatial state $\left|\eta_{i}\right\rangle$ are represented as

$$
\begin{gathered}
\mathcal{E}_{\lambda_{i}}(r, z)=\sqrt{I\left(\lambda_{i}, r, z\right)} H\left(r-\delta_{i-1}^{(\lambda)}\right) H\left(\delta_{i}^{(\lambda)}-r\right), \\
\mathcal{E}_{\eta_{i}}(r, z)=\sqrt{I_{0}(r, z)} H\left(r-\delta_{i-1}^{(\eta)}\right) H\left(\delta_{i}^{(\eta)}-r\right),
\end{gathered}
$$

where $H(r)$ is the Heaviside step function $H(r)=1$ if $r>0$ and is zero otherwise, $\delta_{i}^{(\lambda)}=r_{\lambda_{i}}+\Delta_{i}^{(\lambda)} / 2$ and $\delta_{i}^{(\eta)}=\eta_{i} r_{\max }+$ $\Delta_{i}^{(\eta)} / 2$ for $i=1,2, \ldots, n-1, \delta_{0}^{(\lambda)}=\delta_{1}^{(\lambda)}-\Delta_{1}^{(\lambda)}$, and $\delta_{0}^{(\eta)}=$ $\delta_{1}^{(\eta)}-\Delta_{1}^{(\eta)}$; here, $\Delta_{i}^{(\lambda)}\left(\Delta_{i}^{(\eta)}\right)$ for $i=1,2, \ldots, n-1$ is the distance between the positions of two adjacent spectral (spatial) states, and $\Delta_{i-1}^{(\lambda)}=\Delta_{i}^{(\lambda)}\left(\Delta_{i-1}^{(\eta)}=\Delta_{i}^{(\eta)}\right)$ for $i=1$ and $n$, so that distributions of spectral (spatial) states are nonoverlapping with each other. Both sets of spectral and spatial states are orthogonal, $\left\langle\lambda_{i} \mid \lambda_{j}\right\rangle=\delta_{i j}$ and $\left\langle\eta_{i} \mid \eta_{j}\right\rangle=\delta_{i j}$, where $\delta_{i j}$ is the Kronecker delta. The inner product of two states is given 


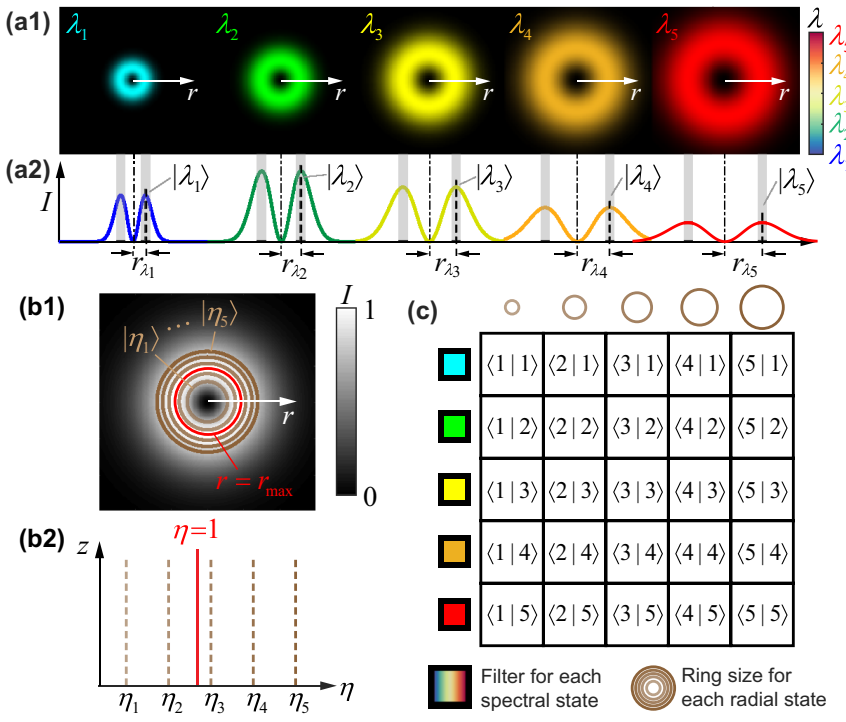

FIG. 4. (a1) and (a2) Procedure for experimental determination of spectral states $\left|\lambda_{i}\right\rangle(i=1,2, \ldots, 5)$ : (a1) The transverse intensity profiles corresponding to different monochromatic components can be obtained by capturing an image of the pulse after propagation through spectral filters at selected wavelengths $\lambda_{i}$. (a2) The radial distribution of the intensity patterns presented in (a1). The radius $r_{\lambda_{i}}$ marks the position at which the intensity of the monochromatic component of wavelength $\lambda_{i}$ reaches its maximum value. (b1) and (b2) Procedure for experimental determination of spatial states $\left|\eta_{i}\right\rangle$ $(i=1,2, \ldots, 5)$ : (b1) The captured transverse profile of the total electric field intensity with the marked position of the recorded radius of $r=r_{\max }(\eta=1)$ at which the total intensity reaches its maximum. Based on the recorded position of $\eta=1$, the positions of various spatial $\left(\left|\eta_{i}\right\rangle\right)$ states can be recorded through radially scaling the position of $\eta=1$ by corresponding ratios of $\eta_{i}$. (b2) Spatial states represented by trajectories in the $\eta-z$ plane. As the trajectories of the spatial states are parallel to the $z$ axis in the $\eta-z$ map, each position of the spatial state can be determined by the corresponding normalized radius $\eta_{i}=r / r_{\max }$. (c) Measurement matrix of state tomography of space-spectrum entangled states, where the inner products are noted as $\langle i \mid j\rangle=\left\langle\eta_{i} \mid \lambda_{j}\right\rangle(i, j=1,2, \ldots, 5)$. The values of $\eta_{i}$ are selected so that the measurement matrix is diagonal for the ideal isodiffracting pulses. Here, the sample number is set to 5 for spectral and spatial states for illustration purposes.

by $\left\langle\eta_{i} \mid \lambda_{j}\right\rangle=\int \mathcal{E}_{\eta_{i}} \mathcal{E}_{\lambda_{j}}^{*} d r$. Here, the definitions of the classical fields $\mathcal{E}_{\lambda_{i}}, \mathcal{E}_{\eta_{i}}$ have been introduced with respect to the radial coordinate $r$ in order to clarify the experimental process for their retrieval. Equivalent definitions can be obtained in terms of the normalized radial coordinate $\eta$ by substituting $r=\eta r_{\max }$.

The classical fields of spectral and spatial states can be experimentally retrieved as follows. For a given spectral state $\left|\lambda_{i}\right\rangle$, the transverse profile of the monochromatic field propagating through a filter at the corresponding wavelength $\lambda_{i}$ can be recorded by a CCD camera at a given propagation distance $z$ [see Fig. 4(a1)]. This allows us to retrieve the peak position of the corresponding intensity $r_{\lambda_{i}}$ and calculate the field function by Eq. (3) [Fig. 4(a2)]. For a spatial state $\left|\eta_{i}\right\rangle$, we should record the total intensity pattern (in the absence of spectral filters) at a propagation distance $z$, which allows us to obtain the total intensity peak position $r_{\max }$ [see Fig. 4(b1)]. The corresponding field profile for the spatial state at $r=\eta_{i} r_{\text {max }}$ can then be calculated by Eq. (4). The values of $\eta_{i}$ are selected with reference to a perfectly isodiffracting pulse (such as the FD), so that the inner product $\left\langle\eta_{i} \mid \lambda_{j}\right\rangle$ is nonzero only if $i=j$ in this ideal STNS case.

Based on the above picture, the STNS is successfully translated into the nonseparability of spectral and spatial states, which resembles the nonseparability of entanglement. In quantum mechanics, there are plenty of mature techniques to quantitatively measure the nonseparability of various kinds of high-dimensional entangled states, such as spin-to-orbital angular momentum entanglement [73], energy-to-time entanglement [74], and radial position-to-momentum entanglement [75]. Based on the analogous mathematical description and physical origin, we introduce the new concept of a spacespectrum nonseparable state that allows us to quantitatively describe pulses with prescribed STNS, such as the FD, i.e., $|\psi\rangle=\sum_{i=1}^{n} c_{i}\left|\eta_{i}\right\rangle\left|\lambda_{i}\right\rangle$, where $c_{i}^{2}=\left\langle\eta_{i} \mid \lambda_{i}\right\rangle$ and $\sum_{i} c_{i}^{2}=1$. On the other hand, for a general pulse, the space-spectrum state is expressed as $|\psi\rangle=\sum_{i=1}^{n} \sum_{j=1}^{n} c_{i, j}\left|\eta_{i}\right\rangle\left|\lambda_{j}\right\rangle$, where $c_{i, j}^{2}=\left\langle\eta_{i} \mid \lambda_{j}\right\rangle$ and $\sum_{i j} c_{i j}^{2}=1$. Experimentally, spatiotemporal pulses can be precisely described by such states with a sufficiently large number $n$ of measurements. We note that here we consider classical broadband beams and pulses as pure states. Our approach can be readily expanded to mixed states, by examining, e.g., pairs or triads of beams and pulses separated in space and/or time (akin to the implementation of the mixed state in prior classical nonseparable modes [76]).

\section{QUANTUM-ANALOGOUS MEASUREMENT}

In this section, we provide the technical details of measuring the STNS with the assistance of the numerical simulations. In analogy with quantum state tomography, we can perform tomography measurements of the space-spectrum state of a spatiotemporal pulse, as Fig. 4(c) shows. Based on the definition of the spectral and spatial states adopted here, the tomography matrix for an isodiffracting pulse, such as the FD pulse, should be diagonal, as shown in Fig. 5(a), revealing space-spectrum entanglement. Importantly, the tomography matrix for isodiffracting pulses is diagonal at any transverse plane; that is, it is propagation invariant. For comparison, we emulate a hypothetical experimentally generated FD pulse by adding noise (see Note 2 of the Supplemental Material [72]) into the ideal FD pulse and calculate the corresponding tomography matrix as shown in Fig. 5(b). Here, the presence of off-diagonal elements indicates that the spectral and radial states are slightly separated and that the pulse indeed deviates from the ideal one. On the other hand, for a wideband LG beam without isodiffraction, the tomography results are propagation dependent. In this case, we average the tomography matrices [Fig. 5(c)] evaluated at various propagation distances [Fig. 5(d)]. The tomography matrices evaluated at different transverse planes, as well as the averaged matrix, are nondiagonal indicating substantial deviation from isodiffracting propagation. Thus the state tomography method introduced here allows us to distinguish the type of STNS in broadband light fields. Indeed, both the FD pulse and the wideband LG beam have degrees of STNS to some extent; however, 

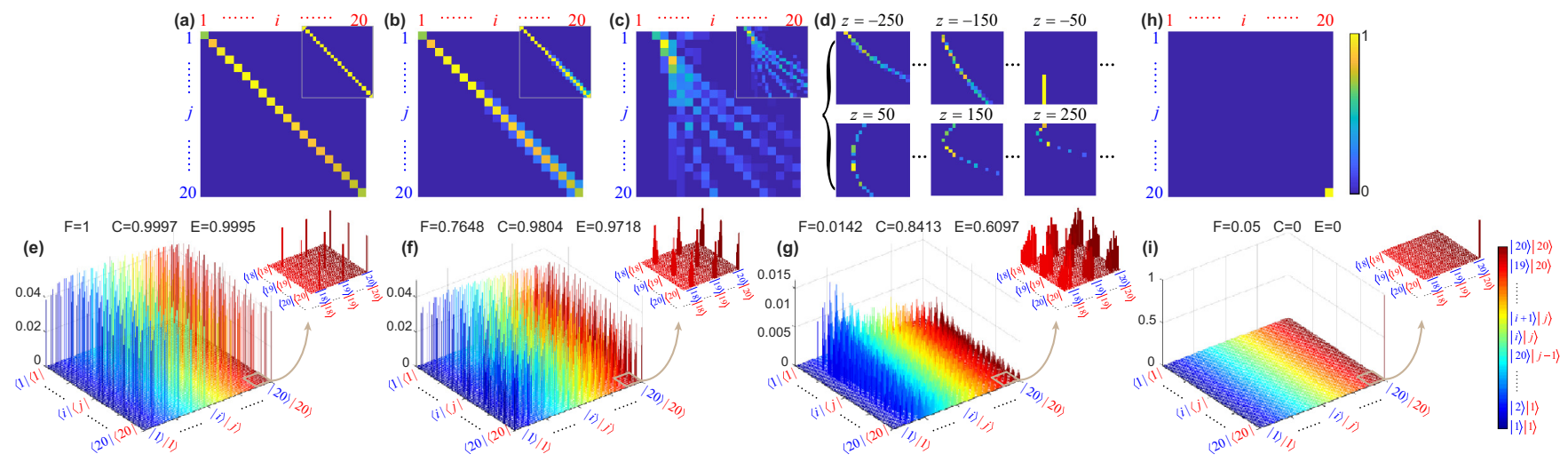

FIG. 5. (a)-(c) The numerical results of quantum-analogous state tomography of the ideal FD pulse (a), the FD pulse with noise (b), and the wideband LG beam (c). The insets at the top right of (a)-(c) are the corresponding results of the intensity-normalized measurements. In (c), the tomography matrix is obtained by averaging multiple measurements at various propagation distances from $z=-250$ to $z=250$ with a step of 50 (unit: $q_{1}$ for FD and $z_{0}$ for LG). (d) The selected tomography matrices of the wideband LG beam at distances of $z= \pm 250, \pm 150, \pm 50$ (unit: $\left.z_{0}\right)$. (e)-(g) The reconstructed density matrices for the ideal FD pulse (e), the FD pulse with noise (f), and the wideband LG beam (g), with marked values of fidelity, concurrence, and EoF, respectively. (h) and (i) The tomography matrix (h) and density matrix (i) for a monochromatic beam. In all panels, red and blue indices, $i$ and $j$, denote spatial $\left|\eta_{i}\right\rangle$ and spectral states $\left|\lambda_{j}\right\rangle$. See the full data set of tomography and density matrices of the wideband LG beam at various propagation distances in Note 3 of the Supplemental Material [72].

only in the case of FD, the STNS leads to isodiffracting propagation.

From the evaluated state tomography matrices, we can reconstruct the corresponding density matrices of the spacespectrum state, $\widetilde{\varrho}=|\widetilde{\psi}\rangle\langle\widetilde{\psi}|$ (where $|\widetilde{\psi}\rangle$ is the measured state). Results for the ideal FD, FD with noise, and wideband LG pulses are listed in Figs. 5(e)-5(g), respectively. Importantly, knowledge of the density matrix allows us to apply quantum measures, e.g., fidelity and concurrence $[42,43]$, to quantitatively characterize the properties of the pulse.

Fidelity. In quantum mechanics, the fidelity is a measure of similarity of two quantum states, defined as $F=$ $\left(\operatorname{Tr} \sqrt{\sqrt{\rho_{1}} \rho_{2} \sqrt{\rho_{1}}}\right)^{2}$, where $\rho_{1}$ and $\rho_{2}$ are the density matrices of the two states. If the target state is a pure state $\left|\psi_{1}\right\rangle$, the density matrix is given by $\rho_{1}=\left|\psi_{1}\right\rangle\left\langle\psi_{1}\right|$, and the fidelity is simplified to $F=\operatorname{Tr}\left(\rho_{1} \rho_{2}\right)=\left\langle\psi_{1}\left|\rho_{2}\right| \psi_{1}\right\rangle$ [42]. Here, we set the target state as the ideal FD pulse $|\psi\rangle=\sum_{i=1}^{n} c_{i}\left|r_{i}\right\rangle\left|\lambda_{i}\right\rangle$. The fidelity of a measured state can then be calculated as $F=$ $\langle\psi|\widetilde{\varrho}| \psi\rangle$, where $\widetilde{\varrho}$ is the density matrix of the measured state. In our implementation, fidelity can quantitatively measure the degree of similarity to an ideal FD pulse taking values from 0 to 1 . The result for the FD with noise is $F=0.7648$, which indicates a high degree of similarity to the ideal FD, while in the case of the wideband LG beam, fidelity approaches zero, $F=0.0142$. Fidelity can be readily defined with respect to different reference pulses (e.g., linearly polarized STNS "focused pancakes" [31]).

Concurrence. In quantum mechanics, the concurrence is a continuous measure of nonseparability of two-dimensional entangled states [42]. This concept was also generalized for high-dimensional cases, usually called $I$ concurrence, defined by $C=\sqrt{2\left[1-\operatorname{Tr}\left(\rho_{A}^{2}\right)\right]}$, where $\rho_{A}$ is the reduced density matrix [77]. For an arbitrary $d$-dimensional state, the concurrence is usually normalized as $C / v_{d}$ and takes values from 0 to 1 $\left[v_{d}=\sqrt{2(1-1 / d)}\right]$, indicating the absence of entanglement (or pure separability) and strong nonseparability (maximum entanglement), respectively. In our study, we use $d=20$ corresponding to the 20 spectral and spatial states. The results for the ideal FD, FD with noise, and wideband LG beam are $C=0.9997, C=0.9804$, and $C=0.8413$, correspondingly. The FD pulse exhibits strong STNS with near-maximum "entanglement," while the wideband LG beam also exhibits a substantial degree of STNS upon propagation owing to the mixing of the different monochromatic components.

Entanglement of formation. In quantum mechanics, the entanglement of formation (EoF) is also a commonly encountered measure of quantum entanglement. EoF is calculated by the von Neumann entropy of the reduced density matrix $E=$ $-\operatorname{Tr}\left[\rho_{A} \log _{2}\left(\rho_{A}\right)\right]$ and is typically normalized as $E / \log _{2}(d)$ in the $d$-dimensional case [78]. In contrast to concurrence, EoF is more sensitive to strong nonseparability due to the convexity of entropic measures. The results for the ideal FD, FD with noise, and wideband LG beam are $E=0.9995, E=0.9718$, and $E=0.6097$, respectively. The lower EoF of the wideband LG beam unveils that it exhibits weaker STNS. We note here that both EoF and concurrence quantify the degree of nonseparability; yet the choice between the concurrence and EoF can be informed by the specific application at hand: EoF (concurrence) is better suited to distinguish between pulses with strong (weak) STNS (see Note 4 of the Supplemental Material [72] for an example explanation).

We note that the ideal FD pulse exhibits very high values of concurrence and EoF (0.9997 and 0.9995), which indicates that the FD is a near-maximally entangled state. Here, the small deviation of the entanglement measures' values from unity is a result of different intensity levels at different spatial and spectral states. However, depending on the problem at hand, we can focus only on the positions of the spatial and spectral states omitting the effects of different intensity levels for different states; then we could regard the FD pulse as a perfect maximally entangled state. In such a case, we can use an intensity-normalized calculation of the inner product $\left\langle\eta_{i} \mid \lambda_{j}\right\rangle=\int \mathcal{E}_{\eta_{i}} \mathcal{E}_{\lambda_{j}}^{*} d r /\left(\int\left|\mathcal{E}_{\eta_{i}}\right| d r \int\left|\mathcal{E}_{\lambda_{j}}\right| d r\right)$ 
TABLE I. Parameter comparison of various kinds of pulse. Fid., fidelity; Conc., concurrence; N-Fid., N-Conc., and N. EoF, fidelity, concurrence, and EoF in intensity-normalized measurement, respectively; W. LG, wideband LG beam; M. LG, monochromatic LG beam.

\begin{tabular}{lccccc}
\hline \hline Pulse & Fid. & Conc. & EoF & N-Fid. & N-Conc. \\
\hline Ideal FD & 1 & 0.9997 & 0.9995 & 1 & 1 \\
Noised FD & 0.7648 & 0.9804 & 0.9718 & 0.7533 & 0.9779 \\
W. LG & 0.0142 & 0.8413 & 0.6097 & 0.0110 & 0.8541 \\
M. LG & 0.0410 & 0 & 0 & 0.0500 & 0 \\
\hline \hline
\end{tabular}

during state tomography. For the ideal FD pulse, the intensitynormalized measurement results in an identity tomography matrix, while fidelity, concurrence, and EoF are all unity. The results of this modified measurement for the ideal FD, the FD with noise, and the wideband LG beam are inserted in Figs. 5(a)-5(c), correspondingly.

As an extreme case of a space-time separable wave, we consider a monochromatic LG beam. The corresponding results of tomography and density matrix are presented in Figs. 5(h) and 5(i), exhibiting only a single nonzero element. As a separable state, it can be expressed in the form $\left|\eta_{n}\right\rangle\left|\lambda_{n}\right\rangle$, resulting in null values of concurrence and EoF. We summarize the results of fidelity and entanglement measures in Table I, for both normalized and non-normalized intensity measurements.

It is worth noting that we do not consider the quantum aspects of the electromagnetic fields, but exploit the mathematical tools of quantum measures to describe classical structured light. Akin to the quantum measures in describing the correlations between two entangled particles, the quantumlike measures introduced here also have clear physical meaning related to the propagation dynamics of spatiotemporal pulses. In particular, the density matrix contains the full information of the correlation between the spectral and spatial states, i.e., the spatial distribution of the monochromatic components of the pulse within the transverse profile of the pulse. The fidelity quantifies the similarity between two pulses. On the other hand, concurrence and EoF quantify the overlap (i.e., correlation) of spectral states (wavelength components) with the spatial states.

\section{DISCUSSION}

We have established a toolkit of quantum-analogous methods to effectively characterize STNS in general electromagnetic beams or pulses, which can not only evaluate the type, but also quantify the strength of the nonseparability. The approach is straightforward and can be easily applied to experimental measurements. Measures such as fidelity, concurrence, and EoF borrowed from quantum mechanics can fully quantify the STNS of an general pulse. In this paper, we consider the FD pulse and a superposition of LG beams as examples for illustration purposes. Nevertheless, with an appropriate modification of spatial states (selection of radial ratios $\eta$ ) our method is readily applicable to a wider class of pulses with arbitrary spatiotemporal structure, such as vector vortex beams [12], pancakelike pulses [31], and isodiverging pulses [14].

Quantitative measures for the characterization of spatiotemporal pulses are crucial for a number of applications. For instance, fidelity allows us to readily evaluate the quality of experimentally generated beams and pulses and also to establish effective criteria for optimizing the corresponding generation schemes. Quantum-analogous measures, such as concurrence and entanglement of formation, may result in translation of quantum techniques (e.g., quantum protocols) in the classical domain for encoding information into structured pulses leading to novel optical communication and cryptography schemes.

While here we focus on fidelity, concurrence, and EoF, a much wider set of quantities has been developed to measure the purity and quality of quantum states, e.g., linear entropy, negativity, the Bell parameter, and the GreenbergerHorne-Zeilinger parameter, to name a few. Hence this set can be mined to further characterize broadband electromagnetic waves with space-time or even more exotic forms of nonseparability. For example, the linear entropy $S=1-\operatorname{Tr}\left(\rho^{2}\right)$, where $\rho=\sum_{j} p_{j}\left|\psi_{j}\right\rangle\left\langle\psi_{j}\right|$ is the density matrix of a measured state and $p_{j}$ is the coefficient of $j$ th mixed state, quantifies how close a quantum state is to a pure $(S=0)$ or a maximally mixed $(S \rightarrow 1)$ state [79]. In this paper, we only consider waves that are represented by pure states; thus the linear entropy of such waves should always be 0 . However, the linear entropy would be very useful, if we consider systems comprising multiple beams. Such a case would be of great interest as we can use the entropy of sets of multiple pulsed beams to encode information, enabling novel applications in high-capacity and encrypted communications by the STNS of pulses. Finally, here we have focused on intensity-only STNS effects; however, the method can be readily extended to include phase effects.

Space-time nonseparable pulses provide unusual and largely unexplored degrees of freedom in structuring light that are yet to be exploited. As such, there is growing interest in the generation and control of high-quality space-time nonseparable pulses. Our method provides the practical quantitative tools for the generation design, optimization, characterization, and detection of such complex pulses, as well as for the study of their light-matter interactions. These key capabilities for taming and exploiting spatiotemporally structured pulses will lead to novel applications in ultrahigh-capacity communications, high-security encryption, topology- and quantum-analogous systems, and metrology that require the manipulation of an increasing number of degrees of freedom. Moreover, of particular interest will be the extension of our work to a unified framework to describe both classical and quantum nonseparability (entanglement), as well as their interplay and connection.

The data from this paper can be obtained from the University of Southampton ePrints research repository [80]. 


\section{ACKNOWLEDGMENTS}

Yijie Shen would like to thank Isaac Nape, Bienvenu Ndagano, Andrew Forbes, H. Esat Kondakci and Ayman F. Abouraddy for useful discussions. The authors acknowledge the support of the MOE Singapore (Grant No. MOE2016T3-1-006), the U.K. Engineering and Physical Sciences
Research Council (Grant No. EP/M009122/1, funder ID given in Ref. [81]), the European Research Council (Advanced Grant No. FLEET-786851, funder ID given in Ref. [82]), and the Defense Advanced Research Projects Agency (DARPA) under the Nascent Light Matter Interactions program.
[1] A. M. Shaltout, K. G. Lagoudakis, J. van de Groep, S. J. Kim, J. Vučković, V. M. Shalaev, and M. L. Brongersma, Spatiotemporal light control with frequency-gradient metasurfaces, Science 365, 374 (2019).

[2] L. Zhang, X. Q. Chen, S. Liu, Q. Zhang, J. Zhao, J. Y. Dai, G. D. Bai, X. Wan, Q. Cheng, G. Castaldi, V. Galdi, and T. J. Cui, Space-time-coding digital metasurfaces, Nat. Commun. 9, 4334 (2018).

[3] Z. Qiao, Z. Wan, G. Xie, J. Wang, L. Qian, and D. Fan, Multivortex laser enabling spatial and temporal encoding, PhotoniX 1, 13 (2020).

[4] Z. Nie, C.-H. Pai, J. Hua, C. Zhang, Y. Wu, Y. Wan, F. Li, J. Zhang, Z. Cheng, Q. Su, S. Liu, Y. Ma, X. Ning, Y. He, W. Lu, H.-H. Chu, J. Wang, W. B. Mori, and C. Joshi, Relativistic single-cycle tunable infrared pulses generated from a tailored plasma density structure, Nat. Photonics 12, 489 (2018).

[5] P. Hilz, T. M. Ostermayr, A. Huebl, V. Bagnoud, B. Borm, M. Bussmann, M. Gallei, J. Gebhard, D. Haffa, J. Hartmann, T. Kluge, F. H. Lindner, P. Neumayr, C. G. Schaefer, U. Schramm, P. G. Thirolf, T. F. Rösch, F. Wagner, B. Zielbauer, and J. Schreiber, Isolated proton bunch acceleration by a petawatt laser pulse, Nat. Commun. 9, 423 (2018).

[6] C. Kerse, H. Kalaycioğlu, P. Elahi, B. Çetin, D. K. Kesim, Ö. Akçaalan, S. Yavaş, M. D. Aşık, B. Öktem, H. Hoogland, R. Holzwarth, and F. Ö. Ilday, Ablation-cooled material removal with ultrafast bursts of pulses, Nature (London) 537, 84 (2016).

[7] E. H. Penilla, L. F. Devia-Cruz, A. T. Wieg, P. Martinez-Torres, N. Cuando-Espitia, P. Sellappan, Y. Kodera, G. Aguilar, and J. E. Garay, Ultrafast laser welding of ceramics, Science $\mathbf{3 6 5}$, 803 (2019).

[8] M. Malinauskas, A. Žukauskas, S. Hasegawa, Y. Hayasaki, V. Mizeikis, R. Buividas, and S. Juodkazis, Ultrafast laser processing of materials: from science to industry, Light: Sci. Appl. 5, e16133 (2016).

[9] Y. Shen, G. Gao, Y. Meng, X. Fu, and M. Gong, Gain-phase modulation in chirped-pulse amplification, Phys. Rev. A 96, 043851 (2017).

[10] L. Rego, K. M. Dorney, N. J. Brooks, Q. L. Nguyen, C.-T. Liao, J. San Román, D. E. Couch, A. Liu, E. Pisanty, M. Lewenstein, L. Plaja, H. C. Kapteyn, M. M. Murnane, and C. Hernández-García, Generation of extreme-ultraviolet beams with time-varying orbital angular momentum, Science 364, eaaw9486 (2019).

[11] K. M. Dorney, L. Rego, N. J. Brooks, J. San Román, C.-T. Liao, J. L. Ellis, D. Zusin, C. Gentry, Q. L. Nguyen, J. M. Shaw, A. Picón, L. Plaja, H. C. Kapteyn, M. M. Murnane, and C. Hernández-García, Controlling the polarization and vortex charge of attosecond high-harmonic beams via simultaneous spin-orbit momentum conservation, Nat. Photonics 13, 123 (2019).
[12] Y. Shen, X. Wang, Z. Xie, C. Min, X. Fu, Q. Liu, M. Gong, and $\mathrm{X}$. Yuan, Optical vortices 30 years on: OAM manipulation from topological charge to multiple singularities, Light: Sci. Appl. 8 90 (2019).

[13] B. E. A. Saleh and M. C. Teich, Fundamentals of Photonics (Wiley, New York, 2019).

[14] M. A. Porras, Diffraction effects in few-cycle optical pulses, Phys. Rev. E 65, 026606 (2002).

[15] M. A. Porras, Characterization of the electric field of focused pulsed Gaussian beams for phase-sensitive interactions with matter, Opt. Lett. 34, 1546 (2009).

[16] D. Hoff, M. Krüger, L. Maisenbacher, A. M. Sayler, G. G. Paulus, and P. Hommelhoff, Tracing the phase of focused broadband laser pulses, Nat. Phys. 13, 947 (2017).

[17] H. E. Kondakci and A. F. Abouraddy, Diffraction-free pulsed optical beams via space-time correlations, Opt. Express $\mathbf{2 4}$ 28659 (2016).

[18] M. Yessenov, B. Bhaduri, H. E. Kondakci, M. Meem, R. Menon, and A. F. Abouraddy, Non-diffracting broadband incoherent space-time fields, Optica 6, 598 (2019).

[19] H. E. Kondakci and A. F. Abouraddy, Diffraction-free spacetime light sheets, Nat. Photonics 11, 733 (2017).

[20] B. Bhaduri, M. Yessenov, and A. F. Abouraddy, Anomalous refraction of optical spacetime wave packets, Nat. Photonics 14 416 (2020).

[21] A. Baltuška, Th. Udem, M. Uiberacker, M. Hentschel, E. Goulielmakis, Ch. Gohle, R. Holzwarth, V. S. Yakovlev, A. Scrinzi, Th. W. Hänsch, and F. Krausz, Attosecond control of electronic processes by intense light fields, Nature (London) 421, 611 (2003).

[22] R. J. Gordon and V. J. Barge, Effect of the Gouy phase on the coherent phase control of chemical reactions, J. Chem. Phys. 127, 204302 (2007).

[23] F. Langer, M. Hohenleutner, C. P. Schmid, C. Pöllmann, P. Nagler, T. Korn, C. Schüller, M. S. Sherwin, U. Huttner, J. T. Steiner, S. W. Koch, M. Kira, and R. Huber, Lightwavedriven quasiparticle collisions on a subcycle timescale, Nature (London) 533, 225 (2016).

[24] J. N. Brittingham, Focus waves modes in homogeneous Maxwell's equations: Transverse electric mode, J. Appl. Phys. (Melville, NY) 54, 1179 (1983).

[25] R. W. Ziolkowski, Exact solutions of the wave equation with complex source locations, J. Math. Phys. (Melville, NY) 26, 861 (1985)

[26] R. W. Ziolkowski, Localized transmission of electromagnetic energy, Phys. Rev. A 39, 2005 (1989).

[27] R. Donnelly and R. Ziolkowski, A method for constructing solutions of homogeneous partial differential equations: Localized waves, Proc. R. Soc. London, Ser. A 437, 673 (1992). 
[28] R. Donnelly and R. W. Ziolkowski, Designing localized waves, Proc. R. Soc. London, Ser. A 440, 541 (1993).

[29] H. E. Hernández-Figueroa, M. Zamboni-Rached, and E. Recami, Localized Waves (Wiley, New York, 2008).

[30] H. E. Hernández-Figueroa, M. Zamboni-Rached, and E. Recami, Non-diffracting Waves (Wiley, New York, 2013).

[31] S. Feng, H. G. Winful, and R. W. Hellwarth, Spatiotemporal evolution of focused single-cycle electromagnetic pulses, Phys. Rev. E 59, 4630 (1999).

[32] R. W. Hellwarth and P. Nouchi, Focused one-cycle electromagnetic pulses, Phys. Rev. E 54, 889 (1996).

[33] K. V. Baryshnikova, D. A. Smirnova, B. S. Luk'yanchuk, and Y. S. Kivshar, Optical anapoles: Concepts and applications, Adv. Opt. Mater. 7, 1801350 (2019).

[34] V. Savinov, N. Papasimakis, D. P. Tsai, and N. I. Zheludev, Optical anapoles, Commun. Phys. 2, 69 (2019).

[35] A. Zdagkas, N. Papasimakis, V. Savinov, M. R. Dennis, and N. I. Zheludev, Singularities in the flying electromagnetic doughnuts, Nanophotonics 8, 1379 (2019).

[36] T. Raybould, V. Fedotov, N. Papasimakis, I. Youngs, and N. Zheludev, Focused electromagnetic doughnut pulses and their interaction with interfaces and nanostructures, Opt. Express 24, 3150 (2016).

[37] T. Kaelberer, V. A. Fedotov, N. Papasimakis, D. P. Tsai, and N. I. Zheludev, Toroidal dipolar response in a metamaterial, Science 330, 1510 (2010).

[38] N. Papasimakis, V. A. Fedotov, V. Savinov, T. A. Raybould, and N. I. Zheludev, Electromagnetic toroidal excitations in matter and free space, Nat. Mater. 15, 263 (2016).

[39] N. Papasimakis, T. Raybould, V. A. Fedotov, D. P. Tsai, I. Youngs, and N. I. Zheludev, Pulse generation scheme for flying electromagnetic doughnuts, Phys. Rev. B 97, 201409(R) (2018).

[40] O. Quevedo-Teruel, H. Chen, A. Díaz-Rubio, G. Gok, A. Grbic, G. Minatti, E. Martini, S. Maci, G. V. Eleftheriades, M. Chen, N. I. Zheludev, N. Papasimakis, S. Choudhury, Z. A. Kudyshev, S. Saha, H. Reddy, A. Boltasseva, V. M. Shalaev, A. V. Kildishev, D. Sievenpiper et al., Roadmap on metasurfaces, J. Opt. 21, 073002 (2019).

[41] R. Horodecki, P. Horodecki, M. Horodecki, and K. Horodecki, Quantum entanglement, Rev. Mod. Phys. 81, 865 (2009).

[42] D. F. V. James, P. G. Kwiat, W. J. Munro, and A. G. White, On the measurement of qubits, in Asymptotic Theory of Quantum Statistical Inference: Selected Papers (World Scientific, Singapore, 2005), pp. 509-538.

[43] E. Toninelli, B. Ndagano, A. Vallés, B. Sephton, I. Nape, A. Ambrosio, F. Capasso, M. J. Padgett, and A. Forbes, Concepts in quantum state tomography and classical implementation with intense light: A tutorial, Adv. Opt. Photonics 11, 67 (2019).

[44] T. Konrad and A. Forbes, Quantum mechanics and classical light, Contemp. Phys. 60, 1 (2019).

[45] E. Karimi and R. W. Boyd, Classical entanglement? Science 350, 1172 (2015).

[46] X.-F. Qian, B. Little, J. C. Howell, and J. H. Eberly, Shifting the quantum-classical boundary: Theory and experiment for statistically classical optical fields, Optica 2, 611 (2015).

[47] A. Aiello, F. Töppel, C. Marquardt, E. Giacobino, and G. Leuchs, Quantum-like nonseparable structures in optical beams, New J. Phys. 17, 043024 (2015).

[48] A. Forbes, A. Aiello, and B. Ndagano, Classically entangled light, Prog. Opt. 64, 99 (2019).
[49] Y. F. Chen, Y. P. Lan, and K. F. Huang, Observation of quantumclassical correspondence from high-order transverse patterns, Phys. Rev. A 68, 043803 (2003).

[50] C. C. Chen, Y. T. Yu, R. C. C. Chen, Y. J. Huang, K. W. Su, Y. F. Chen, and K. F. Huang, Transient Dynamics of Coherent Waves Released from Quantum Billiards and Analogous Observation from Free-Space Propagation of Laser Modes, Phys. Rev. Lett. 102, 044101 (2009).

[51] Y. Shen, X. Yang, X. Fu, and M. Gong, Periodic-trajectorycontrolled, coherent-state-phase-switched, and wavelengthtunable $\mathrm{SU}(2)$ geometric modes in a frequency-degenerate resonator, Appl. Opt. 57, 9543 (2018).

[52] Y. Shen, X. Fu, and M. Gong, Truncated triangular diffraction lattices and orbital-angular-momentum detection of vortex SU(2) geometric modes, Opt. Express 26, 25545 (2018).

[53] Y. Shen, X. Yang, D. Naidoo, X. Fu, and A. Forbes, Structured ray-wave vector vortex beams in multiple degrees of freedom from a laser, Optica 7, 820 (2020).

[54] K. H. Kagalwala, G. Di Giuseppe, A. F. Abouraddy, and B. E. A. Saleh, Bell's measure in classical optical coherence, Nat. Photonics 7, 72 (2013).

[55] L. W. Clark, N. Schine, C. Baum, N. Jia, and J. Simon, Observation of Laughlin states made of light, Nature (London) $\mathbf{5 8 2}$, 41 (2020).

[56] S.-L. Liu, Q. Zhou, S.-K. Liu, Y. Li, Y.-H. Li, Z.-Y. Zhou, G.-C. Guo, and B.-S. Shi, Classical analogy of a cat state using vortex light, Commun. Phys. 2, 75 (2019).

[57] R. C. Devlin, A. Ambrosio, N. A. Rubin, J. P. B. Mueller, and F. Capasso, Arbitrary spin-to-orbital angular momentum conversion of light, Science 358, 896 (2017).

[58] M. McLaren, T. Konrad, and A. Forbes, Measuring the nonseparability of vector vortex beams, Phys. Rev. A 92, 023833 (2015).

[59] D. Giovannini, J. Romero, J. Leach, A. Dudley, A. Forbes, and M. J. Padgett, Characterization of High-Dimensional Entangled Systems via Mutually Unbiased Measurements, Phys. Rev. Lett. 110, 143601 (2013).

[60] A. Forbes and I. Nape, Quantum mechanics with patterns of light: Progress in high dimensional and multidimensional entanglement with structured light, AVS Quantum Sci. 1, 011701 (2019).

[61] H. E. Kondakci, M. A. Alonso, and A. F. Abouraddy, Classical entanglement underpins the invariant propagation of spacetime wave packets, Opt. Lett. 44, 2645 (2019).

[62] D. Guzman-Silva, R. Brüning, F. Zimmermann, C. Vetter, M. Gräfe, M. Heinrich, S. Nolte, M. Duparré, A. Aiello, M. Ornigotti, and A. Szameit, Demonstration of local teleportation using classical entanglement, Laser Photonics Rev. 10, 317 (2016).

[63] B. Ndagano, B. Perez-Garcia, F. S. Roux, M. McLaren, C. Rosales-Guzman, Y. Zhang, O. Mouane, R. I. HernandezAranda, T. Konrad, and A. Forbes, Characterizing quantum channels with non-separable states of classical light, Nat. Phys. 13, 397 (2017).

[64] B. Ndagano, I. Nape, M. A. Cox, C. Rosales-Guzman, and A. Forbes, Creation and detection of vector vortex modes for classical and quantum communication, J. Lightwave Technol. 36, 292 (2017).

[65] A. Sit, F. Bouchard, R. Fickler, J. Gagnon-Bischoff, H. Larocque, K. Heshami, D. Elser, C. Peuntinger, K. Günthner, 
B. Heim, C. Marquardt, G. Leuchs, R. W. Boyd, and E. Karimi, High-dimensional intracity quantum cryptography with structured photons, Optica 4, 1006 (2017).

[66] V. D'ambrosio, N. Spagnolo, L. Del Re, S. Slussarenko, Y. Li, L. C. Kwek, L. Marrucci, S. P. Walborn, L. Aolita, and F. Sciarrino, Photonic polarization gears for ultra-sensitive angular measurements, Nat. Commun. 4, 2432 (2013).

[67] F. Töppel, A. Aiello, C. Marquardt, E. Giacobino, and G. Leuchs, Classical entanglement in polarization metrology, New J. Phys. 16, 073019 (2014).

[68] S. Berg-Johansen, F. Töppel, B. Stiller, P. Banzer, M. Ornigotti, E. Giacobino, G. Leuchs, A. Aiello, and C. Marquardt, Classically entangled optical beams for high-speed kinematic sensing, Optica 2, 864 (2015).

[69] S. Feng, H. G. Winful, and R. W. Hellwarth, Gouy shift and temporal reshaping of focused single-cycle electromagnetic pulses, Opt. Lett. 23, 385 (1998).

[70] A. Zdagkas, N. Papasimakis, V. Savinov, and N. I. Zheludev, Space-time nonseparable pulses: Constructing isodiffracting donut pulses from plane waves and single-cycle pulses, Phys. Rev. A 102, 063512 (2020).

[71] S. Feng and H. G. Winful, Spatiotemporal structure of isodiffracting ultrashort electromagnetic pulses, Phys. Rev. E 61, 862 (2000)

[72] See Supplemental Material at http://link.aps.org/supplemental/ 10.1103/PhysRevResearch.3.013236 for details of the wideband LG beam construction and the tomography and density matrices of the wideband LG beam at various propagation distances, along with other information.

[73] J. Liu, I. Nape, Q. Wang, A. Vallés, J. Wang, and A. Forbes, Multidimensional entanglement transport through single-mode fiber, Sci. Adv. 6, eaay0837 (2020).

[74] J.-P. W. MacLean, J. M. Donohue, and K. J. Resch, Direct Characterization of Ultrafast Energy-Time Entangled Photon Pairs, Phys. Rev. Lett. 120, 053601 (2018).

[75] L. Chen, T. Ma, X. Qiu, D. Zhang, W. Zhang, and R. W. Boyd, Realization of the Einstein-Podolsky-Rosen Paradox Using Radial Position and Radial Momentum Variables, Phys. Rev. Lett. 123, 060403 (2019).

[76] S. M. Hashemi Rafsanjani, M. Mirhosseini, O. S Magana-Loaiza, and R. W. Boyd, State transfer based on classical nonseparability, Phys. Rev. A 92, 023827 (2015).

[77] P. Rungta, V. Bužek, C. M. Caves, M. Hillery, and G. J. Milburn, Universal state inversion and concurrence in arbitrary dimensions, Phys. Rev. A 64, 042315 (2001).

[78] W. K. Wootters, Entanglement of formation and concurrence, Quantum Inf. Comput. 1, 27 (2001).

[79] N. A. Peters, T.-C. Wei, and P. G. Kwiat, Mixed-state sensitivity of several quantum-information benchmarks, Phys. Rev. A 70, 052309 (2004).

[80] https://doi.org/10.5258/SOTON/D1726.

[81] http://dx.doi.org/10.13039/501100000266.

[82] http://dx.doi.org/10.13039/501100000781. 